\title{
Suppression effects on musical and verbal memory
}

\author{
ZACHARY A. SCHENDEL \\ Ohio State University, Columbus, Ohio \\ AND \\ Caroline Palmer \\ McGill University, Montreal, Quebec, Canada
}

\begin{abstract}
Three experiments contrasted the effects of articulatory suppression on recognition memory for musical and verbal sequences. In Experiment 1, a standard/comparison task was employed, with digit or note sequences presented visually or auditorily while participants remained silent or produced intermittent verbal suppression (saying "the") or musical suppression (singing "la"). Both suppression types decreased performance by equivalent amounts, as compared with no suppression. Recognition accuracy was lower during suppression for visually presented digits than during that for auditorily presented digits (consistent with phonological loop predictions), whereas accuracy was equivalent for visually presented notes and auditory tones. When visual interference filled the retention interval in Experiment 2, performance with visually presented notes but not digits was impaired. Experiment 3 forced participants to translate visually presented music sequences by presenting comparison sequences auditorily. Suppression effects for visually presented music resembled those for digits only when the recognition task required sensory translation of cues.
\end{abstract}

The experiments reported here investigated whether the properties of working memory for verbal material also apply to working memory for musical material. Some have proposed separate working memory processes for music (Berz, 1995; Pechmann \& Mohr, 1992). Others have proposed that similar working memory processes account for many types of auditory information, including musical tones (Elliott \& Cowan, 2005; Jones \& Macken, 1993; Salame \& Baddeley, 1989). We addressed this question in three experiments by applying a well-known verbal working memory paradigm to music: the articulatory suppression paradigm.

The articulatory suppression paradigm requires participants to articulate, such as producing the word "the" repeatedly, while viewing and/or rehearsing a stimulus sequence (Murray, 1967, 1968; Saito, 1997, 1998; Salame \& Baddeley, 1989). Murray's (1967, 1968) experiments indicated that recall of visually presented letter sequences during articulatory suppression was impaired, relative to a no-suppression control condition (Murray, 1967). In other experiments (Murray, 1968), the phonological similarity effect (recalling phonologically dissimilar sequences more accurately than phonologically similar ones) occurred only while participants engaged in articulatory suppression during auditory presentation of letter sequences; the phonological similarity effect was abolished during visual presentation. Murray (1968) reasoned that auditorily stored information that gains access to the phonological store is more prone to interference by acoustic similarity than is information from the visual modality.

Experiments employing other forms of suppression have since replicated Murray's $(1967,1968)$ findings. Saito (1997, 1998) had participants engage in intermittent suppression (repeating the word "ah") or continuous suppression ("ahhhhhh"). The phonological similarity effect was abolished only in the intermittent suppression condition (Saito, 1998), which led Saito to conclude that any intermittent articulation would cause interference with verbal rehearsal. When a whistling suppression task (both intermittent and continuous) was substituted for the "ah" task, only the intermittent whistle suppression reduced the phonological similarity effect (Saito, 1998).

Several experiments have investigated whether impaired memory for verbal material in the presence of suppression is due to general interference or, more specifically, to the use of articulators. Secondary tasks that do not use articulators, such as tapping a steady rhythm, listening to a voice (Gupta \& MacWhinney, 1995), perceptuomotor tracking, or visual pattern recall (Cocchini, Logie, Della Sala, MacPherson, \& Baddeley, 2002), do not interfere with verbal working memory as severely as does suppression. Conversely, rhythmically clicking one's teeth, silent or vocalized articulatory suppression, lip-synching to recorded auditory interference, or intermittent whistling result in greater memory decrements in recall of verbal material (Gupta \& MacWhinney, 1995; Saito, 1998).

C.Palmer, caroline.palmer@mcgill.ca 
Baddeley and Hitch (1994) interpreted Murray's (1967, 1968) and other articulatory suppression findings in terms of the phonological loop component of their working memory model. The phonological loop specializes in working memory for auditory information with two components: the phonological store, which stores active information, and an articulatory control process, which is responsible for rehearsal and recoding (Baddeley, 1990). Information in the phonological store decays in approximately $2 \mathrm{sec}$ (Baddeley, 1986) and must be rehearsed with the articulatory control process in order to remain active. Auditory verbal material has direct access to the phonological store, whereas visually presented verbal material must be translated into an auditory form by the articulatory control process. Baddeley and Hitch (1994) concluded that articulatory suppression interacts with stimulus modality because it prevents translation of visual information into an auditory form.

Although much evidence has accumulated in support of the phonological loop, it is not universally accepted. In other models (Brown, Preece, \& Hulme, 2000; Nairne, 1990 ), forgetting is accounted for solely by interference. In the feature model (Nairne, 1990; Nairne \& Kelly, 1999), memory traces are represented by feature vectors, and memory relies on the availability of retrieval cues; the feature model distinguishes between modality-independent features (in an abstract representation) and modalitydependent features (those related to presentation conditions, including presentation modality) and assumes that they do not interfere with each other. Auditory traces are thought to have more modality-dependent features than do visual traces. Articulatory suppression effects are accounted for in terms of reducing the distinctiveness of modality-independent features (due to the encoded features of the articulated item); auditory traces are retrieved more successfully because they have more modalitydependent features (Neath \& Nairne, 1995). Other work suggests that the effects of suppression depend on the articulatory complexity of suppression, leading some to question whether those effects indicate speech production mechanisms, rather than phonological memory (Jones, Macken, \& Nicholls, 2004). These alternative depictions, however, do not make specific predictions for memory for music versus speech.

\section{Working Memory for Music}

Berz (1995) argued that working memory for musical material could operate independently of working memory for verbal material. He proposed an additional slave system that encodes music in a separate working memory store. Berz's main source of evidence for an additional musical loop was the irrelevant sound experiments of Salame and Baddeley (1989), who presented different background material to participants: pink noise, Arabic speech, instrumental music, and vocal music. The more speech-like the background stimulus, the more it interfered with memorization of digit sequences. One explanation proposed by the authors was that the more acoustic features the background material had in common with speech, the more direct access it had to the phonological store and, thus, the more interference it created.

Only a few studies have directly compared the relationship between working memory for language and music (Deutsch, 1970; Logie \& Edworthy, 1986; Pechmann \& Mohr, 1992). Logie and Edworthy conducted an articulatory suppression experiment with memory for tones. Recognition accuracy for standard/comparison tones was impaired by articulatory suppression and by a homophone judgment task (e.g., plain-plane), but not by visual symbol matching. Impaired recognition during articulatory suppression and homophone judgments was interpreted as being a result of articulatory interference, attributed to subvocal speech/singing, and not of interference due to general cognitive load. Deutsch employed a standard/comparison paradigm with single musical tones and interpolated verbal or musical interference. Participants showed decreased accuracy for interpolated tones, in comparison with interpolated digits, suggesting domainspecific interference. Pechmann and Mohr extended Deutsch's paradigm to other interpolated conditions between the standard/comparison tones: attended verbal, unattended verbal, attended visual, unattended visual, and tonal conditions. Musically trained participants showed results similar to those in Deutsch: significant interference only in the interpolated tone condition.

There are important distinctions between memory studies with musical and verbal materials that may influence comparisons. Whereas the majority of studies of verbal memory use recall tasks, studies of musical memory rely on recognition tasks (because people cannot verbalize tones easily, and the method of recall on a musical instrument is not well specified in the auditory signal). Second, the musical memory tasks tend to rely on recognition of single tones, which do not tax memory load or contain much order information or opportunity for interference from other stimulus items, all of which are known to influence memory for verbal materials (i.e., Cowan, Saults \& Nugent, 2001; Jones, Farrand, Stuart, \& Morris, 1995) and musical materials (Palmer \& Pfordresher, 2003; Roberts, 1986). In the experiments reported here, short sequences of musical and verbal materials were used in a recognition paradigm, to facilitate comparisons.

In three experiments, we investigated whether the articulatory suppression and presentation modality effects in working memory for verbal materials would be mirrored in working memory for music. Each experiment used a standard/comparison recognition task with four-item digit or musical note sequences, paired with verbal or musical intermittent suppression or with visual interference. Participants sang the nonsense syllable "la" (commonly used in vocal exercises) repeatedly on a particular musical pitch; this musical suppression was designed to be comparable to (verbal) articulatory suppression from repeating "the" (Murray, 1967; Saito, 1997). If rehearsal of verbal and musical material requires similar auditory memory mechanisms, musical and verbal suppression should equivalently impair memory for digits and tones. If rehearsal of verbal and musical material entails differ- 
ent memory mechanisms, the suppression type should differentially impair memory for digits and tones.

\section{EXPERIMENT 1}

Experiment 1 employed a standard/comparison forced choice procedure with self-produced intermittent suppression. The standard and comparison sequences contained four digits or musical notes, presented either visually or auditorily. Musically experienced participants engaged in verbal suppression, musical suppression, or no suppression during stimulus presentation and during a retention interval. Verbal suppression consisted of producing "the" at a constant rate; musical suppression consisted of singing "la" at a constant rate on a constant pitch. We examined whether visually presented stimuli would be impaired more by suppression than were auditorily presented stimuli, following Murray (1967, 1968), and whether verbal and musical suppression would cause differential interference to each type of stimulus material. Differential effects of verbal and melodic suppression on digits and tones were examined within participants, to control for any individual differences in listeners' musical expertise that might influence working memory for auditory sequences.

\section{Method}

Participants. Forty-eight English-speaking adults (16-23 years old) from Columbus, Ohio, with at least 6 years of private lessons on a musical instrument $(M=7.92$ years, range $=6-16$ years $)$, participated in the study. The 24 participants assigned to the auditory presentation condition had an amount of musical training ( $M=8.0$ years) equivalent to that for those assigned to the visual presentation condition ( $M=7.8$ years). Only participants who completed correctly a music notation task designed to ensure that they could read notes on a treble clef staff were included.

Materials and Apparatus. Fifty-three 4-item melodic sequences were randomly generated from the nine pitches in the $\mathrm{C}$ Major diatonic scale (from D4 to E5), with no successive repeated pitches or melodic intervals equal to or greater than an octave. Comparison sequences were the same as the standard sequences half of the time, and they differed by one note (a whole or a half step) half of the time. Half of the pitch changes occurred in the second position of the 4 -item sequence, and half occurred in the third position. Half of the changes ascended in pitch, and half descended in pitch. The visually presented notes appeared as stemless quarter notes that moved vertically in the same location on a motionless treble clef musical staff. The auditory tones were sampled at $44 \mathrm{kHz}$ with a piano timbre, using Cakewalk Professional, with a duration (from onset to offset) of $450 \mathrm{msec}$. The tone sequences were created by splicing together single recordings of each tone with 700-msec interonset intervals (IOIs) to avoid possible timbral variations.

Fifty-three 4-item digit sequences were randomly generated from the digits 1-9 in a similar fashion, with no successive repeated digits. Comparison sequences were the same as the standard sequences on half of the trials, and they differed by one digit on the other half All changes were of a one-digit magnitude (e.g., 6-7 or 6-5). The visually presented digits appeared at the same location on the screen as the visual tones, in the middle of a box whose outline was equal to the size of the musical staff. The auditory digit sequences were created from a recording of a female voice speaking the digits 1 through 9. The duration (from onset to offset) of the digits was $400 \mathrm{msec}$ (the slightly shorter duration of the spoken digits than of the musical tones was judged more natural). The digit sequences were created by splicing together the recordings of the single digits with 700msec IOIs.

Stimulus trials were constructed with a visually orienting “+” appearing on the computer screen for $1,000 \mathrm{msec}$, followed by a fouritem standard sequence presented at the rate of one item $/ 700 \mathrm{msec}$. Following the disappearance of the last standard item, a 4,200-msec retention pause occurred; this was followed by a second 700 -msec visually orienting "+" indicating the onset of the comparison sequence, also displayed at one item/700 msec. The spoken digits and computergenerated piano tones were standardized to the same maximum sound level. All stimuli were presented on a PC with Presentation software through AKG headphones. The participants wore a head-mounted microphone to record their suppression voicings directly to a Tascam DA-30 DAT machine (44 kHz/sec sampling rate).

Design. A 2 (presentation modality: visual or auditory) $\times 3$ (suppression type: verbal, musical, or none) $\times 2$ (stimulus type: digits or notes) mixed factorial design was used in Experiment 1. Presentation modality was a between-participants factor, following Murray (1967); all other factors were within participants. Suppression type was blocked, and stimulus type was blocked within the suppression blocks. The order of suppression blocks and stimulus type was counterbalanced across participants. Each participant received 12 no-suppression trials, 16 verbal suppression trials, and 16 musical suppression trials for each of the two stimulus types and 3 practice trials in each of six conditions, totaling 106 trials in all.

Procedure. The participants completed the music notation task first. Then they were seated in front of the display monitor and were given three practice trials at the beginning of each block. At the beginning of each suppression trial, the participants heard a recording of a male voice saying "the" or a recording of a male voice singing "la" on C4 (not one of the stimulus tones) to indicate the suppression pitch they should use. The participants were told to speak "the" or to sing "la" to coincide with the onset of each stimulus item after the first orienting "+." The participants were required to continue suppression during the retention interval at the same rate until they saw the second "+." Then the four-item comparison sequence was presented. The participants indicated whether the sequences were the same or different by pressing the corresponding labeled key on a keyboard. Halfway through the experiment, the participants completed a musical experience questionnaire. The entire experiment lasted $1 \mathrm{~h}$.

\section{Results}

The participants' suppression IOIs were measured to assess their produced suppression rate. Sixteen participants, chosen at random for the analysis, showed no significant differences between verbal suppression IOIs $(M=667.23 \mathrm{msec})$ and musical suppression IOIs $(M=$ $665.78 \mathrm{msec})$.

The participants' mean accuracy scores (percent correct) are shown in Figure 1 by presentation modality and stimulus type in the three suppression conditions. First, we will report analyses that compare suppression conditions with no-suppression control conditions; then we will examine suppression effects in the absence of control conditions. A 3 (suppression type) $\times 2$ (stimulus type) $\times 2$ (presentation modality) ANOVA on the accuracy scores indicated a main effect of suppression type $\left[F(2,92)=55.9, M S_{\mathrm{e}}=0.0218\right.$, $p<.01]$, a main effect of stimulus type $[F(1,46)=269.52$, $\left.M S_{\mathrm{e}}=0.0108, p<.01\right]$ and a significant interaction of suppression type with stimulus type $[F(1,46)=17.75$, $\left.M S_{\mathrm{e}}=0.0138, p<.01\right]$. The difference between digit and note performance was smaller during no-suppression control trials ( $8 \%$ difference in accuracy) than during verbal 

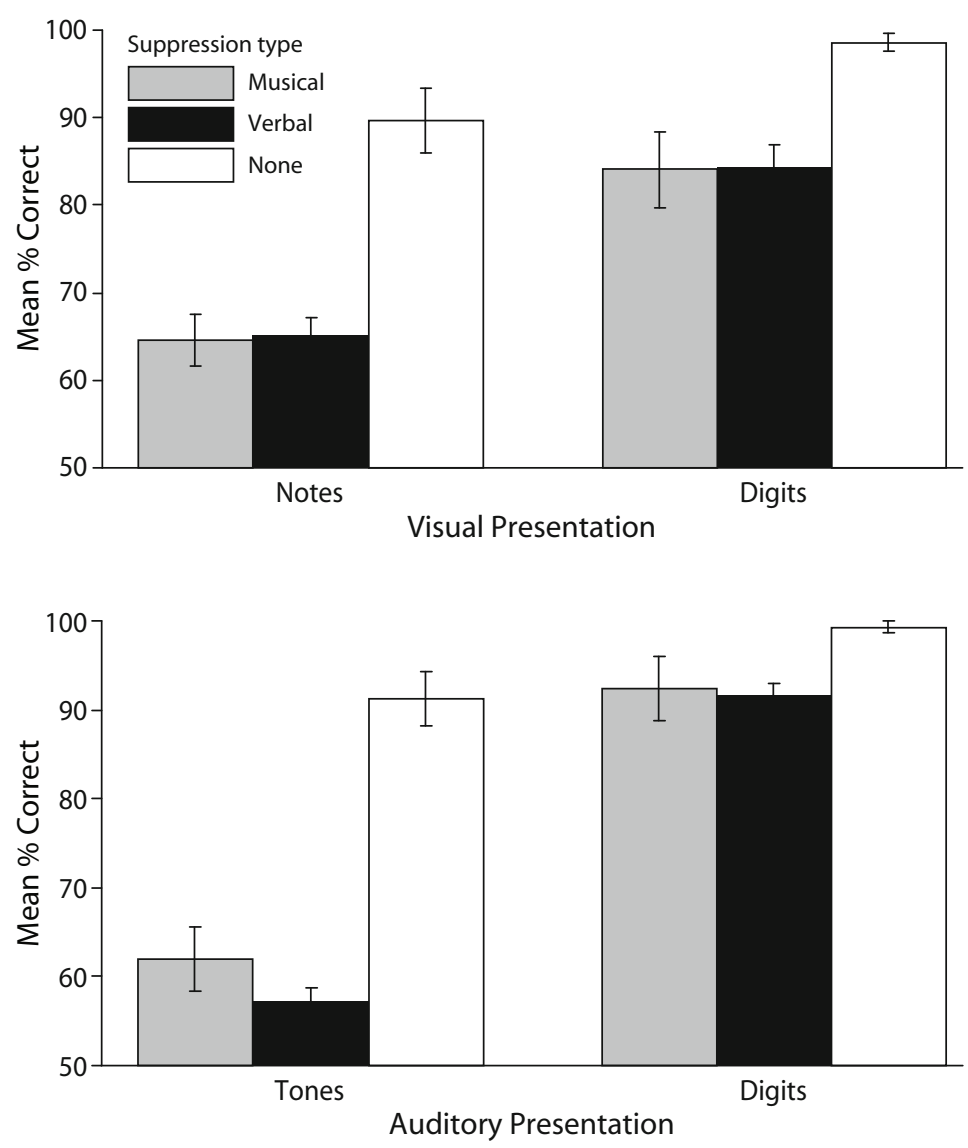

Figure 1. Experiment 1: Mean recognition accuracy (with standard error bars) for digits and notes by presentation modality (visual or auditory) and suppression type (verbal, musical, or none).

suppression (25\% difference) or musical suppression (27\% difference). There was also a significant interaction of stimulus type with presentation modality $[F(1,46)=11.57$, $\left.M S_{\mathrm{e}}=0.0108, p<.01\right]$. Overall recognition of digits was better in auditory presentation (Tukey HSD $=.0580, p<$ $.05)$; recognition of notes did not differ by modality. The three-way interaction between stimulus type, suppression type, and presentation modality approached significance $\left[F(2,92)=3.06, M S_{\mathrm{e}}=0.0138, p<.10\right]$.

Next, we compared the effects of musical and verbal suppression in the absence of no-suppression control trials. An ANOVA on accuracy scores by suppression type (musical or verbal), stimulus type, and presentation modality indicated no significant differences among suppression types and no interactions of suppression type with other variables. There was a significant main effect of stimulus type $\left[F(1,46)=307.56, M S_{\mathrm{e}}=0.01, p<.01\right]$ and a significant interaction between stimulus type and presentation modality $\left[F(1,46)=19.42, M S_{\mathrm{e}}=0.01\right.$, $p<.01]$. This interaction is shown in Figure 2, collapsed across the suppression conditions. Suppression lowered recognition accuracy for digits in visual presentation relative to auditory presentation, but this was not the case for tones. Planned comparisons confirmed a significant dif- ference between visual and auditory digit presentations with suppression $[t(47)=2.45, p<.05]$ and no significant difference between visual note and auditory tone presentations with suppression.

This analysis was repeated on participants' $d^{\prime}$ scores; a hit was defined as a correct response to a change in the comparison sequence, whereas a correct rejection was defined as a correct response to no change in the comparison sequence. Again, there were no differences between verbal suppression (mean $d^{\prime}=3.66$ ) and musical suppression (mean $d^{\prime}=3.34$ ) or interactions of suppression with other variables. There was a main effect of stimulus type $\left[F(1,46)=215.182, M S_{\mathrm{e}}=3.72, p<.01\right]$ and an interaction of stimulus type with presentation modality $\left[F(1,46)=19.69, M S_{\mathrm{e}}=3.72, p<.01\right]$. Similar to the accuracy scores, planned comparisons confirmed a significant difference between visual and auditory digit presentations with suppression $[t(47)=2.95, p<.05]$ and no significant difference between visual note and auditory tone presentations with suppression.

The participants' musical experience (measured by years of musical training or performing experience) did not correlate with performance in the recognition of tone sequences, either in accuracy scores or $d^{\prime}$ values. 


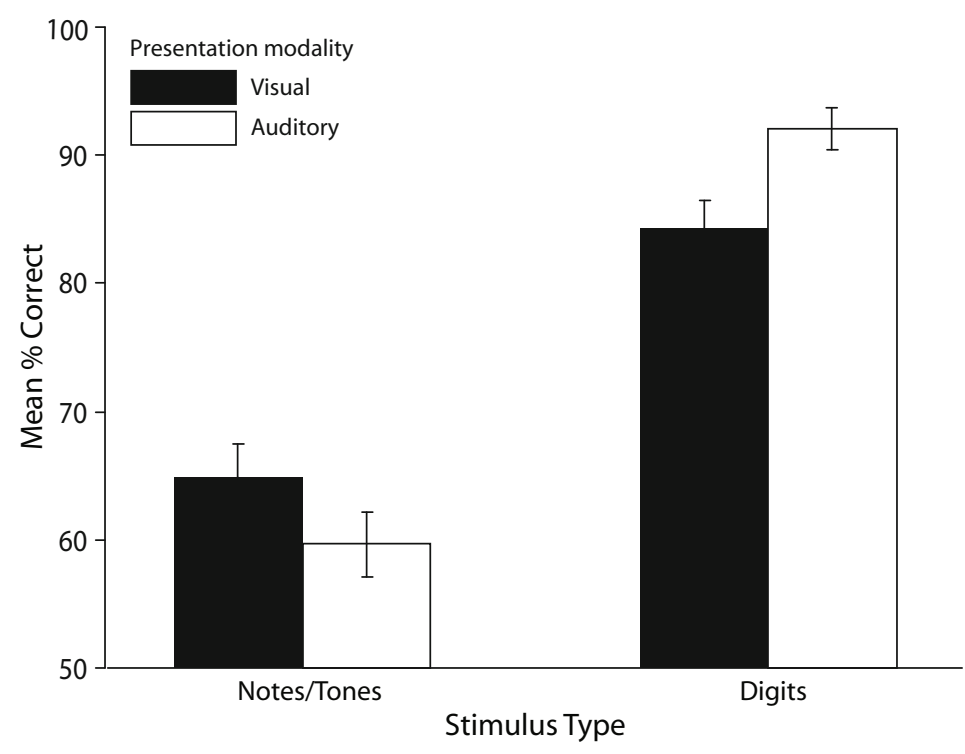

Figure 2. Experiment 1: Mean recognition accuracy (with standard error bars) on suppression trials for digits and notes by presentation modality (visual or auditory).

\section{Discussion}

This experiment yielded several findings. First, musical suppression caused an impairment on recognition accuracy equivalent to that of verbal suppression, both for digits and for notes. It may be that musical and verbal suppression did not differ in how much interference they caused because both suppression types required intermittent use of the articulators, consistent with Saito's (1998) finding that whistling and speaking suppress memory for verbal material equivalently. Second, digits were recognized more accurately than notes overall, which may be due, in part, to the fact that people have more experience memorizing random digit sequences (such as phone numbers) than memorizing random tones. This difference in stimulus accuracy may change with extended practice or musical experience, a possibility we explored in Experiment 3.

Third, verbal suppression impaired musically experienced participants' recognition of digits more during visual presentation than during auditory presentation, consistent with Murray's $(1967,1968)$ findings that support the presence of a phonological loop (Baddeley, 1990). However, musical suppression did not impair note recognition more during visual or auditory presentation. It is important to note that the same participants who showed the traditional articulatory verbal suppression effect exhibited similar impairments of digit recognition during musical suppression, while exhibiting a lack of articulatory suppression in recognition of notes. Thus, the interactions of suppression with presentation modality could not be attributed solely to individual differences in stimulus familiarity that arose with musical experience.

Why suppression did not have the same effect on memory for musical materials-recognition accuracy was slightly (although not significantly) higher for visually presented notes than for auditorily presented notes-may be due to several factors. We assumed that the participants translated the visually presented material into an auditory form for rehearsal. However, visually presented notes could be encoded in many ways, including note names, direction of melodic contour, or spatial coordinates indicated by staff lines and spaces. In principle, visual information would be governed by the visuospatial sketchpad, which is not impaired by articulatory suppression (Cocchini et al., 2002). Visuospatial and other information inherent in visually presented notes may explain the superior recognition accuracy for visual notes over auditory notes in the suppression conditions. Experiment 2 was designed to test this possibility by including a visual interference task instead of suppression.

\section{EXPERIMENT 2}

Experiment 2 incorporated a standard/comparison task with both visual and auditory presentation of digits and notes. Visual interference was introduced during the retention interval. If the retention of to-be-remembered material is aided by the use of visual cues, visual interference should significantly impair performance (Cocchini et al., 2002). We hypothesized that visual interference would interfere with the retention of visual notes but would not interfere with visual digits, auditory tones, or auditory digits.

\section{Methods}

Participants. Sixteen English-speaking adults (18-20 years old) from Columbus, Ohio with at least 6 years of private lessons on a musical instrument or voice $(M=7.97$ years, range $=6-12$ years $)$ participated in the study. No participant in Experiment 2 took part in the previous experiment.

Materials and Apparatus. The same materials and apparatus as those in Experiment 1 were used. An additional 23 digit and 23 note sequences were created, following the same constraints as those 
in Experiment 1 . Twelve unique $4 \times 4$ black-and-white visual interference grids were created by randomly filling 5 of the 16 squares (Pechmann \& Mohr, 1992). The entire area and the placement of the grids were designed to be displayed in the same area as the digits and notes, including the area covered by the musical staff.

Design. A 2 (presentation modality: visual or auditory) $\times 2$ (presence or absence of visual interference) $\times 2$ (stimulus type: digits or notes) within-participants design was used in Experiment 2 . The order of presentation modality blocks was counterbalanced across participants. The order of stimulus blocks and of visual interference blocks was counterbalanced within presentation modality blocks. The participants received 16 trials in each of the eight presentation modality/visual interference/stimulus type conditions, as well as 3 practice trials in each of the eight blocks, totaling 152 trials in all.

Procedure. The trials were the same as those in Experiment 1, with the following exception: During the 4,200-msec retention interval, six visual interference grids were presented for $200 \mathrm{msec}$, each with 700-msec IOIs. The participants were told to immediately indicate with a buttonpress whether the last two grids were the same or different, before the stimulus sameldifferent judgment. Half of the time, the last two grids were the same, and half of the time, they were different. The participants were given three practice trials before each block. The entire experiment lasted $1 \mathrm{~h}$.

\section{Results}

Response accuracy on the intervening visual grid task resulted in a mean of $97.36 \%$ correct, with no significant differences across conditions.

Figure 3 shows the mean recognition accuracy scores in the standard/comparison task. A 2 (presentation modality) $\times 2$ (visual interference) $\times 2$ (stimulus type) ANOVA on the accuracy scores indicated a main effect of stimulus type $\left[F(1,15)=46.17, M S_{\mathrm{e}}=0.0189, p<.01\right]$. There was a main effect of visual interference $[F(1,15)=$ $\left.8.95, M S_{\mathrm{e}}=0.0047, p<.01\right]$ and a significant interaction between visual interference and stimulus modality $\left[F(1,15)=9.48, M S_{\mathrm{e}}=0.0044, p<.01\right]$. The three-way interaction between stimulus type, presentation modality, and presence/absence of visual interference was significant as well $\left[F(1,15)=7.67, M S_{\mathrm{e}}=0.0055, p<.05\right]$. Visual and auditory digit trials showed similar small drops in performance when visual interference was introduced, although both conditions were near ceiling; in contrast, note trials showed a large performance decrease in visual presentation with visual interference and no decrease in
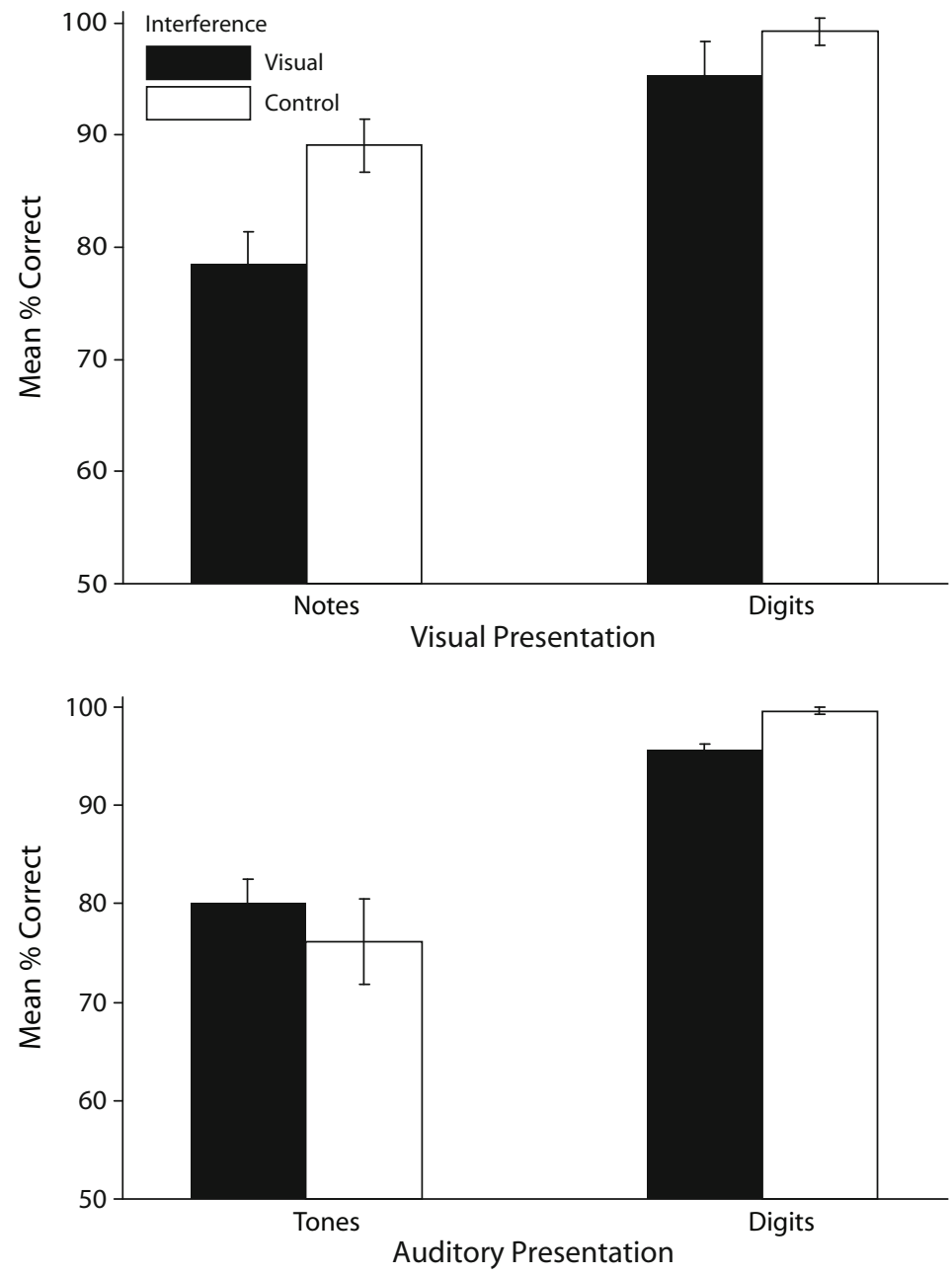

Figure 3. Experiment 2: Mean recognition accuracy (with standard error bars) for digits and notes by presentation modality (visual or auditory) and interference type (visual or none). 
auditory presentation with visual interference. Post hoc comparisons on the means associated with the three-way interaction indicated that the drop in performance when visual interference was introduced was significant only for visually presented notes (Tukey HSD $=.0944, p<$ .01 ), and no other comparisons reached significance. Visual interference selectively impaired memory for visually presented notes.

The same analysis conducted on the participants' $d^{\prime}$ values indicated a main effect of stimulus type $[F(1,15)=$ $\left.76.76, M S_{\mathrm{e}}=4.93, p<.01\right]$ and a main effect of presence/absence of visual interference $[F(1,15)=14.63$, $\left.M S_{\mathrm{e}}=2.17, p<.01\right]$, and the three-way interaction between presentation modality, presence/absence of visual interference, and stimulus type approached significance $\left[F(1,15)=2.92, M S_{\mathrm{e}}=2.77, p=.10\right]$. Again, the drop in sensitivity when visual interference was introduced was largest for visually presented notes $\left(d^{\prime}=4.3\right.$ without visual interference, $d^{\prime}=3.1$ with interference).

The participants' musical experience (years of training or performing experience) did not correlate with performance on the recognition of tone sequences.

\section{Discussion}

Visual interference selectively impaired memory for visually presented notes. Visual interference did not impair the same participants' memory for visually presented digits, consistent with previous findings that visually presented digits are transferred to an auditory or phonological form (Murray, 1967). The differential effects of visual interference suggest that memory for visually presented notes relies, at least partially, on visuospatial information, more so than does memory for visually presented digits or auditorily presented notes or digits. The visual interference task was an attention-demanding secondary task, and the participants were highly accurate at this task in all conditions. Since visual interference selectively impaired visually encoded notes in Experiment 2, this suggests that visually presented notes were not transformed and rehearsed in a purely auditory form. Although suppression and visual interference may not cause equivalent forms of memory impairment, Experiment 2's findings suggest that the drop in performance due to suppression in Experiment 1 was due to the articulatory nature of the suppression, rather than to attentional distraction or general cognitive load.

Thus, the findings of Experiments 1 and 2 indicate that suppression and visual interference differentially reduced recognition accuracy of visually presented notes. Accuracy in the visually presented note control trials (in the absence of suppression or interference) indicated the same level of performance across experiments (Experiment 1, 89\% correct; Experiment 2, 89\% correct), ruling out effects due to participant differences across experiments. If visually presented notes are encoded in a combination of auditory and visual cues, articulatory suppression may affect the auditory cues, whereas visual interference affects the visual cues. Whether participants rely on visual or auditory information to aid memorization may be influenced by the context in which the memorization occurs.
An alternative explanation is that producing intermittent suppression causes more attentional distraction than does passively watching intermittent visual interference; however, this explanation does not account for stimulus modality differences. In Experiment 3, we pursued the question of auditory and visual encoding in memory for musical materials.

\section{EXPERIMENT 3}

Experiment 3 was designed to force the participants to rehearse visually presented music in an auditory form by presenting a visual standard, followed by an auditory comparison, under suppression conditions. This manipulation ensured that extra-auditory cues, although present in the visual standard, could not aid the participants in the recognition task. Experiment 3 was also designed to test the original hypothesis: that visual and auditory presentation of notes are differentially affected by suppression, in a within-participants comparison of presentation modalities. Presentation modality effects in Experiment 1 were evaluated between participants, as in Murray (1967, 1968); although there were no group differences in musical expertise in Experiment 1, individual differences could, in principle, have influenced the presentation modality effects (Elliott \& Cowan, 2005). Therefore, in contrast to Experiment 1, in which the participants were able to rehearse the visually presented music under natural conditions (in whatever manner they preferred), Experiment 3 forced the participants to translate the notation into an auditory form in order to succeed at the task. Musically experienced participants completed the standard/ comparison task with musical notes while producing musical, verbal, or no suppression. On half of the trials, the participants compared two auditory tone sequences (auditoryauditory trials), and on the other half they compared a visual note sequence with an auditory tone sequence (visual-auditory trials). Suppression should differentially impair memory for the visual-auditory trials if the articulatory suppression findings for verbal material (Murray, 1967, 1968) hold in memory for music.

\section{Method}

Participants. Sixteen native English-speaking adult musicians (19-25 years old) from Montreal with at least 6 years of private lessons on a musical instrument or voice $(M=11.41$ years, range $=$ 6-16 years) participated in the experiment.

Materials and Apparatus. In Experiment 3, the same musical materials as those in Experiment 2 were used; all stimuli were music. An additional 30 note sequences were created for this experiment. The participants' suppression voicings were recorded to a Marantz Solid State Recorder PM670 (44 KHz/sec sampling rate).

Design and Procedure. A 2 (presentation modality: visual to auditory or auditory to auditory) $\times 3$ (suppression type: verbal, musical, or none) within-participants design was used in Experiment 3. Presentation modality was blocked, and suppression type was blocked within the presentation blocks. The order of presentation modality blocks and suppression blocks within modality blocks was counterbalanced across participants. The design and procedure were the same as those in Experiment 1, except that on half of the trials, the standard sequence was presented visually and the comparison sequence was presented auditorily; on the other half, an auditory 


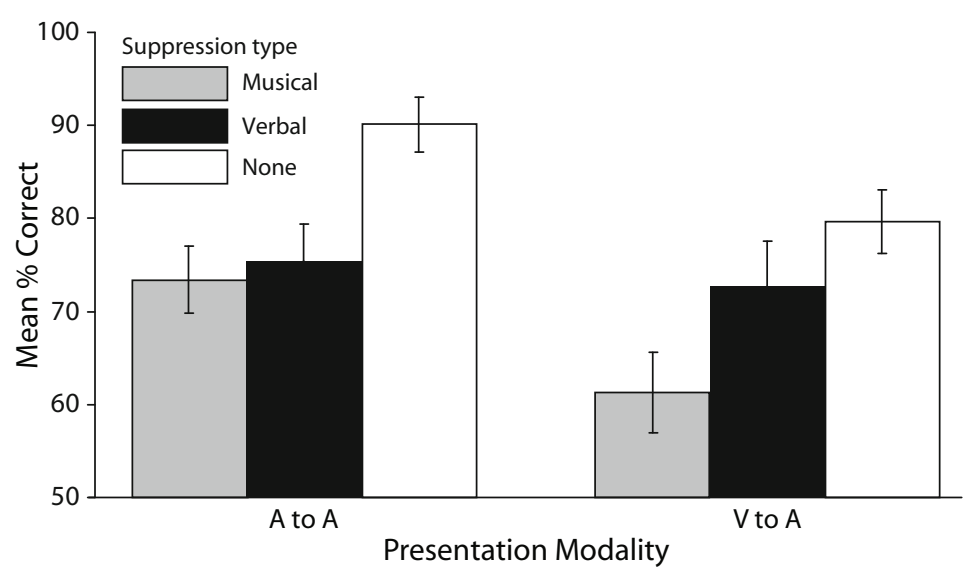

Figure 4. Experiment 3: Mean recognition accuracy (with standard error bars) for notes by presentation modality (visual to auditory [ $\mathrm{V}$ to $\mathrm{A}]$ or auditory to auditory [A to A]) and suppression type (verbal, musical, or none).

standard was followed by an auditory comparison. Each participant received 12 control trials, 16 verbal suppression trials, and 16 musical suppression trials in each of two presentation modalities, plus 5 practice trials in each of six blocks, totaling 118 trials in all.

\section{Results}

The participants' suppression IOIs indicated no differences in suppression rate across conditions. Eight participants, chosen at random for the analysis, indicated equivalent rates for verbal suppression IOIs $(M=687.66 \mathrm{msec})$ and musical suppression IOIs $(M=697.82 \mathrm{msec})$.

The participants' mean accuracy scores are shown in Figure 4. Again, the effects of suppression will first be compared with those in no-suppression control conditions and then will be examined separately from control conditions. A 3 (suppression type: musical, verbal, or no suppression) $\times 2$ (presentation modality) ANOVA on the accuracy scores indicated a main effect of presentation modality $\left[F(1,15)=11.35, M S_{\mathrm{e}}=0.015, p<.01\right]$ and a main effect of suppression type: $[F(2,30)=10.53$, $\left.M S_{\mathrm{e}}=0.0238, p<.01\right]$, and the interaction of suppression type with modality approached significance $[F(2,30)=$ 2.59, $\left.M S_{\mathrm{e}}=0.0077, p=.09\right]$. As is shown in Figure 4, both musical and verbal suppression reduced recognition, relative to no suppression (Tukey $=.0981, p<.05$ ); this finding for auditory-auditory presentation conditions (left half of Figure 4) is the same as that in Experiment 1 (the same conditions shown in the bottom left of Figure 1). Recognition of visual-auditory presentation in suppression conditions, relative to the no-suppression condition, is also reduced, although more by musical suppression than by verbal suppression. Analyses of $d^{\prime}$ values showed similar results: a main effect of presentation modality $\left[F(1,15)=5.84, M S_{\mathrm{e}}=3.74, p<.01\right]$, a main effect of

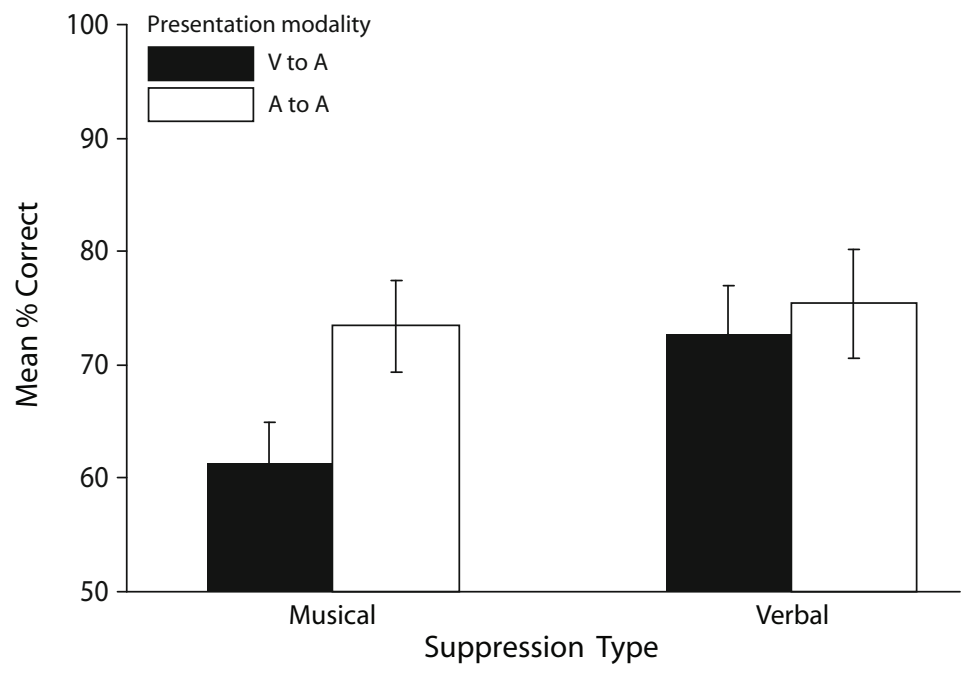

Figure 5. Experiment 3: Mean recognition accuracy (with standard error bars) for notes on suppression trials by presentation modality (visual to auditory [V to A] or auditory to auditory [A to A]). 
suppression type $\left[F(2,30)=8.28, M S_{\mathrm{e}}=6.51, p<.01\right]$, and a significant interaction between presentation modality and suppression type $\left[F(2,30)=3.82, M S_{\mathrm{e}}=1.84, p<\right.$ $.05]$. Sensitivity to auditory-auditory presentation conditions was reduced for both musical and verbal suppression, relative to no suppression; sensitivity to visual-auditory presentation was reduced only for musical suppression, relative to no suppression (Tukey $=1.06, p<.05$ ).

Next, we will compare performance under musical and verbal suppression without the control conditions. Figure 5 shows the results for the different suppression types presented by presentation modality, as in Figure 2 (Experiment 1). An ANOVA on the suppression trials indicated a main effect of presentation modality $[F(1,15)=$ $\left.5.79, M S_{\mathrm{e}}=0.0152, p<.05\right]$ and an interaction with suppression type $\left[F(1,15)=6.14, M S_{\mathrm{e}}=0.0057, p<.05\right]$. Planned comparisons indicated that musical suppression impaired performance more during visual-auditory presentation than during auditory-auditory presentation $[t(15)=3.67, p<.01]$; performance with verbal suppression did not differ between presentation modalities. As is shown in Figure 5, the musical suppression results are similar to Murray's $(1967,1968)$ suppression findings with verbal material: Recognition accuracy during musical suppression was lower for visual-auditory presentation than for auditory-auditory presentation.

The effects of musical experience on recognition memory were evaluated in correlational analyses on the recognition scores with years of performing experience on a musical instrument. There was a negative correlation between participants' decrement in visual note recognition from no-suppression to musical suppression with amount of performing experience $(r=-60, p<.05)$; participants with less musical experience were more impaired by musical suppression on visual-auditory presentation trials. Similar correlations of musical expertise with recognition for auditorily presented notes or with no-suppression minus verbal suppression conditions did not reach significance. When musical experience was treated as a categorical variable of high/low experience based on a median split in an ANOVA, the musical experience factor did not reach significance or interact with any other factors. This analysis was likely influenced by a restricted range of musical experience; the musicians in this experiment were all highly experienced, with 9-22 years of performing experience.

\section{Discussion}

In contrast to the previous experiments, Experiment 3 showed that musical suppression impaired recognition of visually presented notes more than it impaired recognition of auditorily presented tones, but only when the participants were forced to translate visually presented notes into an auditory representation. This finding is consistent with the modality-specific findings of articulatory suppression in memory for digits (Murray, 1967, 1968). This is the first demonstration, to our knowledge, of domain-specific suppression effects on recognition memory for music. This is important because it demonstrates that memory for notes exhibits the same characteristic breakdowns during suppression as memory for digits, but only when musically experienced participants are forced to translate visual presentation into an auditory form.

Memory recognition in the auditory-auditory tone trials in Experiment 3 replicated the findings for auditory tone trials in Experiment 1; verbal and musical suppression caused similar amounts of impairment on these trials. Only visual-auditory tone trials were distinguished in impairment by musical or verbal suppression. This finding is consistent with explanations of forgetting based on the degree of similarity between the stimulus and the interference materials (Nairne, 1990; Salame \& Baddeley, 1989). These findings suggest that verbal and musical suppression may cause similar amounts of interference to auditory presentation of digits and notes, for the reason that both digits and tones are remembered auditorily, whereas visually presented music is not transformed by default, unless participants are forced to transform it to an auditory form. Interestingly, suppression impairment was mediated by amount of musical experience; musically experienced participants were less susceptible to interference from musical suppression for visually presented music than were less experienced participants. This is consistent with the general effect that the ability to form auditory (musical) images tends to increase with musical experience (Aleman, Neiuwenstein, Bocker, \& de Haan, 2000).

\section{GENERAL DISCUSSION}

Three main findings arose from comparisons of recognition memory for verbal and musical material. First, musical and verbal suppression impaired recognition accuracy for both digit and tone sequences, as compared with no suppression. Aside from a single condition (visual-to-auditory presentation of notes), musical and verbal suppression did not differentially affect recognition of digits or tones in Experiment 1 or 3. Second, only visually presented notes were impaired by visual interference, indicating that the participants encoded and/or rehearsed visually presented verbal and musical material in different ways. Finally, memory for musical material was differentially affected by musical suppression in ways consistent with Murray's $(1967,1968)$ findings of articulatory suppression for verbal materials, only when the participants were forced to translate visual material into an auditory form for rehearsal.

Participants in all experiments recognized digit sequences with higher accuracy than note sequences, despite the fact that they had musical training. Although more musical experience or training may improve musical memory, it may never reach the superiority of memory for verbal material; Roberts's (1986) serial recall experiments with highly trained music students at Juilliard also indicated superiority for digit recall over musical tones. Random digit sequences, such as telephone numbers, are more commonly encountered than are random musical tones; superior recognition of random digit sequences (as opposed to tone sequences) may result from experience.

Similar impairment of tone and digit recognition from musical and verbal suppression is consistent with Saito's 
(1998) conclusion that any intermittent use of the articulators (whether singing or speaking) is capable of suppressing memory for auditory material. Saito (1998) concluded that any sound produced intermittently by the articulators (including speaking "la" or whistling) was an effective suppressor. Although speaking "the" repeatedly and singing "la" on a constant pitch did not generate much variation in the suppressant, the materials they suppressed varied substantially in acoustic features, and thus these findings extend Saito's (1998) conclusion to auditory forms of music.

Comparisons between memory for music presented in visual and auditory modalities shed light on how musicians encode notated music. Visual interference impaired retention of visually presented music, consistent with Cocchini et al.'s (2002) findings for recall of visual material. The finding that visual interference significantly impaired recognition of visually presented notes while not having any significant effect on auditory tones, visual digits, or auditory digits indicates that memory for visually presented notes incorporates visuospatial cues; the finding that either type of suppression impaired memory for both visually and auditorily presented music indicates the use of auditory cues as well. Comparisons among impairments from visual interference and suppression suggest that neither visual nor auditory cues alone can account for memory for visually presented music.

Musical experience mediated differences in visual presentation of musical materials; less experienced participants showed more impairment from musical suppression for visually presented music than did more experienced participants. Sloboda (1976) also found differences in recall of visual musical notation due to musical experience; experienced musicians were superior to nonmusicians in recalling brief visual displays of notated music of four or more notes. He hypothesized that experienced musicians were better able to chunk and name musical patterns and that both musicians and nonmusicians rely initially on visual cues but musicians are able to chunk the stimuli in more accurate representations. Reduced impairment from musical suppression is consistent with an improved-chunking interpretation; the ability to form an accurate auditory representation from visual presentation increases with musical experience, a finding documented in many paradigms (Finney \& Palmer, 2003; Highben \& Palmer, 2004). Whether other relevant factors alter with musical experience, such as sensitivity to pitch distinctiveness (Cowan, et al., 2001) or ability to rehearse an auditory image independently of presentation modality (Keller, Cowan, \& Saults, 1995), is not yet known.

The findings of differential memory impairment from suppression when participants were forced to match visual with auditory stimuli is unique and may result from the integration of visual and auditory cues in a single representation. The other experiments demonstrated the use of visual and auditory cues, but not necessarily their integration. How integration occurs across sensory modalities is a matter for debate. On the basis of a visual similarity effect for letters that was exacerbated by articulatory suppression, Logie, Della Sala, Wynn, and Baddeley (2000) concluded that both visual and auditory codes were created. To explain these findings and others, Baddeley (2000) proposed an episodic buffer, which combines information from the phonological loop and the visuospatial sketchpad in a single multidimensional code. Other models (Nairne, 1990) assume a weighting of features across multiple modalities. On the basis of irrelevant sound effects, Jones (1993) proposed a blackboard model in which different sensory stimuli are combined. Although the experiments reported here do not distinguish between these alternatives, they indicate that context variables (the need to translate standard/comparison across modalities) and individual differences (musical experience) influence the weighting of sensory cues in memory tasks

Is memory for music like memory for speech? The intriguing finding that memory for music is differentially susceptible across presentation modalities to auditory interference from suppression suggests a parallel with the phonological loop predictions for verbal memory (Baddeley \& Hitch, 1974). The findings of Experiments 1 and 2 do not, however, support a modular view of working memory that distinguishes between memory for music and speech. These findings are more consistent with evidence of mechanisms common to different types of auditory traces; proposals include general auditory segmentation mechanisms (Bregman, 1990; Jones et al., 1995), retrieval-based features subject to similarity-based interference (Jones \& Macken, 1993; Nairne, 1990), and individual differences in activated features from longterm memory (Cowan, Saults \& Nugent, 1997; Elliott \& Cowan, 2005). The latter explanation, in particular, is consistent with the fact that musical experience mediates the differential interference of suppression on visually presented music.

In sum, although domain-specific storage or rehearsal mechanisms for music (Berz, 1995) are possible, a simpler account of these findings is that the same mechanisms are responsible for the storage and rehearsal of verbal material and auditorily encoded music, at least for participants with sufficient experience in both domains. Individual differences are prominent in memory for visually presented music that includes both visuospatial and auditory features, as is indicated by the visual interference and suppression effects reported here. How the integration of these features changes with musical experience is a topic for future research.

\section{AUTHOR NOTE}

This research was supported by NSERC Grant 298173 to the second author and by the Canada Research Chairs program. We thank Neal Johnson, Dylan Jones, and Mari Riess Jones for comments on an earlier draft and Scott Jankowski, Erik Sullivan, and Nikolai Shatalov for assistance with the experiments. Correspondence may be addressed to $\mathrm{Z}$. A. Schendel (e-mail: schendez@ hotmail.com), or to C. Palmer, Department of Psychology, McGill University, 1205 Dr Penfield Ave., Montreal, QC, H3A 1B1 Canada (e-mail: caroline.palmer@mcgill.ca). 


\section{REFERENCES}

Aleman, A., Neiuwenstein, M. R., Bocker, K. B. E., \& De HaAn, H. F. (2000). Music training and mental imagery ability. Neuropsychologia, 38, 1664-1668.

BADDELEY, A. D. (1986). Working memory. Oxford: Oxford University Press, Clarendon Press.

BADDELEY, A. D. (1990). Human memory: Theory and practice. Hove, U.K.: Erlbaum.

BADDEley, A. D. (2000). The episodic buffer: A new component of working memory? Trends in Cognitive Sciences, 4, 417-423.

Baddeley, A. D., \& Hitch, G. J. (1974). Working memory. In G. H. Bower (Ed.), The psychology of learning and motivation (Vol. 8, pp. 47-89). New York: Academic Press.

BADDELEY, A. D., \& Hitch, G. J. (1994). Developments in the concept of working memory. Neuropsychology, 8, 485-493.

BERz, W. A. (1995) Working memory in music: A theoretical model. Music Perception, 12, 353-364.

Bregman, A. (1990). Auditory scene analysis. Cambridge, MA: MIT Press.

Brown, G. D. A., Preece, T., \& Hulme, C. (2000). Oscillator-based memory for serial order. Psychological Review, 107, 127-181.

Cocchini, G., Logie, R. H., Della Sala, S., MacPherson, S. E., \& BADDEley A. D. (2002). Concurrent performance of two memory tasks: Evidence for domain-specific working memory systems. Memory \& Cognition, 30, 1086-1095.

Cowan, N., Saults, J. S., \& Nugent, L. D. (1997). The role of absolute and relative amounts of time in forgetting within immediate memory: The case of tone-pitch comparisons. Psychonomic Bulletin \& Review, 4, 393-397.

Cowan, N., SAults, J. S., \& Nugent, L. D. (2001). The ravages of absolute and relative amounts of time on memory. In H. L. Roediger III, J. S. Nairne, I. Neath, \& A. Surprenant (Eds.), The nature of remembering: Essays in honor of Robert G. Crowder (pp. 315-330). Washington, DC: American Psychological Association.

Deutsch, D. (1970). Tones and numbers: Specificity of interference in immediate memory. Science, 168, 1604-1605.

Elliott, E. M., \& CowAN, N. (2005). Coherence of the irrelevant-sound effect: Individual profiles of short-term memory and susceptibility to task-irrelevant materials. Memory \& Cognition, 33, 664-675.

FinNey, S. A., \& PALMer, C. (2003). Auditory feedback and memory for music performance: Sound evidence for an encoding effect. Memory \& Cognition, 31, 51-64.

Gupta, P., \& MacWhinney, B. (1995). Is the articulatory loop articulatory or auditory? Reexamining the effects of concurrent articulation on immediate serial recall. Journal of Memory \& Language, 34, 63-88.

Highben, Z., \& PAlmer, C. (2004). Effects of auditory and motor mental practice in memorized piano performance. Bulletin of the Council for Research in Music Education, 159, 58-65.

JONES, D. M. (1993). Objects, threads and streams of auditory attention. In A. Baddeley \& L. Weiskrantz (Eds.), Attention: Selection, awareness, and control (pp. 87-104). Oxford: Oxford University Press, Clarendon Press.

Jones, D. M., Farrand, P., Stuart, G., \& Morris, N. (1995). Func- tional equivalence of verbal and spatial information in serial shortterm memory. Journal of Experimental Psychology: Learning, Memory, \& Cognition, 21, 1008-1018.

Jones, D. M., \& MACKEN, W. J. (1993). Irrelevant tones produce an irrelevant speech effect: Implications for phonological coding in working memory. Journal of Experimental Psychology: Learning, Memory, \& Cognition, 19, 369-381.

Jones, D. M., Macken, W. J., \& Nicholls, A. P. (2004). The phonological store of working memory: Is it phonological and is it a store? Journal of Experimental Psychology: Learning, Memory, \& Cognition, 30, 656-674.

Keller, T. A., Cowan, N., \& Saults, J. S. (1995). Can auditory memory for tone pitch be rehearsed? Journal of Experimental Psychology: Learning, Memory, \& Cognition, 21, 635-645.

Logie, R. H., Della Sala, S., Wynn, V., \& Baddeley, A. D. (2000). Visual similarity effects in immediate verbal serial recall. Quarterly Journal of Experimental Psychology, 53A, 626-646.

Logie, R. H., \& Edworthy, J. (1986). Shared mechanisms in the processing of verbal and musical material. In D. G. Russell, D. F. Marks, \& J. T. E. Richardson (Eds.), Imagery II (pp. 33-37). Dunedin, New Zealand: Human Performance Associates.

Murray, D. J. (1967). The role of speech responses in short-term memory. Canadian Journal of Psychology, 21, 263-267.

MurRaY, D. J. (1968). Articulation and acoustic confusability in shortterm memory. Journal of Experimental Psychology, 78, 679-684.

NAIRNE, J. S. (1990). A feature model of immediate memory. Memory \& Cognition, 18, 251-269.

NaIRne, J. S., \& Kelly, M. R. (1999). Reversing the phonological similarity effect. Memory \& Cognition, 27, 45-53.

Neath, I., \& Nairne, J. S. (1995). Word-length effects in immediate memory; Overwriting trace decay theory. Psychonomic Bulletin \& Review, 2, 429-441.

Palmer, C., \& Pfordresher, P. Q. (2003). Incremental planning in sequence production. Psychological Review, 110, 683-712.

Pechmann, T., \& Mohr, G. (1992). Interference in memory for tonal pitch: Implications for a working-memory model. Memory \& Cognition, 20, 314-320.

RoBERTS, L. A. (1986). Modality and suffix effects in memory for melodic and harmonic materials. Cognitive Psychology, 18, 123-157.

SAITO, S. (1997). When articulatory suppression does not suppress the activity of the phonological loop. British Journal of Psychology, 88, 565-578.

SAITo, S. (1998). Phonological loop and intermittent activity: A whistle task as articulatory suppression. Canadian Journal of Experimental Psychology, 52, 18-23.

Salame, P., \& BADDELEY, A. D. (1989). Effects of background music on phonological short-term memory. Quarterly Journal of Experimental Psychology, 41A, 107-122.

SLoboda, J. A. (1976). Visual perception of musical notation: Registering pitch symbols in memory. Quarterly Journal of Experimental Psychology, 28, 1-16.

(Manuscript received December 21, 2005; revision accepted for publication April 3, 2006.) 